\title{
Severity of malnutrition and treatment responses in under five children in Bahir Dar Felegehiwot Referral Hospital, Northwest Ethiopia
}

\author{
Lijalem Mekonnen, Ahmed Abdusemed, Mulate Abie, Asmare Amuamuta* \\ Bahir Dar University, College of Medicine and Health Sciences, P.O. Box 79, Bahir Dar, Ethiopia \\ Email address: \\ lijalem_mekonin@yahoo.com (L. Mekonnen),daaha223@hotmail.com (A. Abdusemed), kefalebri2006@gmail.com (M. Abie), \\ asmareamu2005@yahoo.com (A. Amuamuta)
}

\section{To cite this article:}

Lijalem Mekonnen, Ahmed Abdusemed, Mulate Abie, Asmare Amuamuta. Severity of Malnutrition and Treatment Responses in under Five Children in Bahir Dar Felegehiwot Referal Hospital, Northwest Ethiopia. Journal of Food and Nutrition Sciences.

Vol. 2, No. 3, 2014, pp. 93-98. doi: 10.11648/j.jfns.20140203.18

\begin{abstract}
Introduction: Malnutrition is one of the leading causes of morbidity and mortality in children under the age of five in developing countries. Ethiopia being one of these countries, malnutrition is an important public health problem. However, little information is available on the severity of malnutrition and treatment responses in under five children especially in hospital settings. Objective: To determine the severity of malnutrition and treatment responses of severe acute malnutrition in children under the age of five and to identify its associated factors. Methodology: ensus method was used and a retrospective data of about 1639 under five children admitted to Felegehiwot Referral Hospital (pediatrics department) form November 2012 -November 2013 were studied for severe acute malnutrition and its treatment responses. Results: In the study period, a total of 1639 children less than 5 years of age were admitted to FHRH pediatric department, and out of that 145 were under five years old with a diagnosis of malnutrition malnourished characteristics. Hence, the proportion of under five aged children affected with malnutrition from this one-year retrospective data in FHRH was about $8.8 \%$. From the total of 145 malnourished children, about 93(64.1\%) were detected for wasting/acute malnutrition and about 36 (24.8\%) were affected with severe wasting or severe acute malnutrition (SAM). Among the malnourished children, about 133(91.7\%) were detected for stunting (chronic malnutrition) and about 65(44.8\%) were affected with severe stunting. Conclusions: The findings of this study showed that the proportion of malnutrition in under five children is high especially during 1-2 years old group. Among the malnourished children detected (145), the level of stunting/chronic malnutrition was higher than wasting/acute malnutrition (91.7\% vs $64.1 \%$, respectively). The level of severe chronic stunting investigated was also found higher than the level of SAM ( $44.8 \%$ vs $24.8 \%$, respectively). Better responses of treatment and condition at discharge were observed by using different therapeutic combinations after admission. To reduce childhood malnutrition especially in under five children, due emphasis should be given in improving the knowledge and practice of parents on appropriate infant and young child feeding practices and treatment service practices from nearby health facilities.
\end{abstract}

Keywords: Malnutrition, Under five Children, Retrospective study, Treatment Response and Condition at Discharge, Felegehiwot Referral Hospital

\section{Introduction}

Hunger and malnutrition remain among the most devastating problems worldwide, particularly facing the worlds under privileged and poor. Malnutrition covers a broad spectrum of illnesses, including under nutrition, specific nutrient deficiencies and over nutrition, and kills, retards, cripples, blinds and impairs human developments on truly massive scale worldwide [1].
The crisis of malnutrition is real, and its persistence has profound and frightening implications for children, society and the future of human kind. Malnutrition is not, as many think, a simple matter of whether a child can satisfy his/her appetite. A child who eats enough to satisfy immediate hunger can still be malnourished. Three quarters of children who died world wide of causes related to malnutrition are 
what nutritionists describe as mildly to moderately malnourished [1].Increased illness often accompanies malnutrition. Morbidity and malnutrition are synergistically related in that malnourished children are more predisposed to illness of infectious origins and ill children are more likely to become malnourished. And many more studies identified that social, cultural and economic characteristics of the community are responsible for child malnutrition [2].

Children are the most nutritionally vulnerable group especially in the developing world. According to surveys conducted between 1987 and 1998, two out of five of these children are stunted, one in three is underweight and one in ten is wasted; the numbers vary considerably among regions. And annually, some 30 million infants, around 82,000 every day, are born with intra uterine growth retardation due to mainly poor nutrition status. The highest levels of stunting are from Eastern Africa, where on average $48.1 \%$ of preschool children are affected in the year 2000. This region includes Ethiopia, where a national survey in 1992 found that $64.2 \%$ of under five children were stunted. In Eastern Africa stunting is increasing at $0.08 \%$ per year $[3,4]$.

Ethiopia is one of the countries in the sub-Saharan Africa with the highest rates of malnutrition. No progress has been made in reducing child malnutrition over the past 17 years. Some of the underlying and basic causes for the problem could be due to low agricultural production, low and inadequate food consumption, diseases and falling Gross National product per capita. Drought, political instability and mass population movement are also the major contributing factors. The problem is deep-rooted in areas of prolonged civil war and drought, where social rehabilitation becomes much more complex due to disruption of social support network, displacement of populations and environmental degradation $[3,5]$.

Ethiopia has consistently been facing severe pre-school childhood malnutrition problems, with malnutrition rates ranking amongst the worst sub-Saharan Africa [6]. According to the house hold survey which was conducted in SNNPRS in 2003, although results show statistically significant improvement in wasting and underweight compared to the 2000 survey, elevated levels of malnutrition were still present, particularly among children 12-23 months of age. The report showed that one in ten children 12-23 months of age were found to suffer from moderate or severe wasting [7].

More surprisingly, a very high prevalence of stunting was observed in the food surplus regions of the country. West Gojjam Zone for example is an area with surplus food production but surprisingly with high prevalence of child malnutrition. According to the 1992 national nutrition survey, the prevalence rate of stunting in children in West Gojjam was $75 \%$ [8].This suggests that food security at regional or zonal level does not necessarily mean nutritional security at individual level. Jemal and Tsegaye [9] also indicated that although food security is necessary, it is not the only determining factor in ensuring adequate nutritionalstatus in the area. Other researchers also recommended that the factors contributing to the highest rate of malnutrition need further investigation.

Despite the existence of various surveys conducted in different areas of our country as mentioned above on malnutrition in children, there exists scarce information on the severity of the problem and treatment responses especially in hospital based study settings. Therefore, this study was designed to determine the severity of malnutrition in under five children who were admitted to FHRH in one year (November 2012- November 2013). In addition, the treatment responses for SAM in under five children in FHRH were also investigated.

\section{Methods}

\subsection{Study Area}

The study was conducted in FHRH, which is the referral hospital for Amhara Regional state, located in the capital city of the region Bahir Dar. The Referal Hospital was founded in 1962 since that time it is has served about 5 million people being as the only referral hospital for many decades. The Pediatric department is among the five inpatient departments in FHRH. Bahir Dar, the next stop 563 kilometers from Addis Ababa is located on the Southern shore of Lake Tana, the source of the Blue Nile.

\subsection{Study Design}

Hospital based retrospective one year study on all under five children admitted with the diagnosis of malnutrition from November 2012-November 2013 was done. Regarding sampling, a census data was used to determine the severity of malnutrition for admitted under five children. Anthropometry which is defined as the scientific study of the measurement and proportions of the human body was used as a technique for assessing the size, proportions and composition of the body [10]. For the purpose of the study, the anthropometric indices used to determine childhood malnutrition were height-for-age (stunting), weight-for- age (underweight) and weight-for-height (wasting).

Acute malnutrition (wasting) is a nutritional deficit state of recent onset related to sudden food deprivation or malabsorption or poor utilization of nutrients which results in rapid weight loss. It refers to low weight-for-height (at $<2$ SD or 70-90 percentile of the median value of the weight -for- height according to the National Center for Health Statistics (NCHS/WHO) international reference [10]. Whereas, severe wasting/SAM is defined by the value of $<3 \mathrm{SD}$ (<70 percentile).Chronic malnutrition (stunting) reflects long-term cumulative effects of inadequate nutrition and health. Stunting is defined as low height-forage (at $<-2$ SD or 85-95 percentile of median value of the NCHS/WHO international growth reference. Severe stunting is defined by the value of $<-3 \mathrm{SD}$ ( $<85$ percentile). Underweight as an anthropometrics index of weight-for age represents body mass relative to age. 
Underweight(using Harvard curve) refers to a deficit and is defined as low weight for age (at <-2 SD or 60-80 percentile of the median value of the NCHS/WHO international reference). Whereas severe underweight is considered for the value of $<3 \mathrm{SD}$ ( $<60$ percentile). Mid upper arm circumference (MUAC) was also measured to determine the degree of malnutrition in under five children.

\subsection{Data Collection Procedure}

Data were collected by using prepared check list that contains demographic factors, clinical presentations, anthropometric values, hematological values (hemoglobin levels and RBC size), mode of management and treatment responses. Patients' chart number was collected from the pediatrics ward registration book and by using these chart numbers, charts were drawn by card room workers.

\subsection{Data Management and Analysis}

After data collection, each filled checklist was checked for completeness. Data was entered with Microsoft excel and analyzed by using descriptive statistics. Percentages, tables and graphs were used to present the results.

\subsection{Ethical Clearance}

Ethical approval was obtained from Institutional Review Board of Bahir Dar University, College of Medicine and Health Sciences. Besides, all the information retrieved was kept in the way that could not interfere in personal confidentiality.

\section{Results}

\subsection{Overall Proportion of Malnutrition and other Characteristics}

In the study period a total of 2192 children less than 14 years of age were admitted to FHRH pediatric department, from which 1639 cases were under five years old and among whom 145 were under five years old children detected with malnutrition. The proportion of under five aged children affected with malnutrition from the one-year retrospective data in FHRH was about $8.8 \%$.

The most common complaints at the time of admission were generalized body swelling (around 30\%) followed by diarrhea (about 23\%) of all the cases (as indicated on Figure 1).

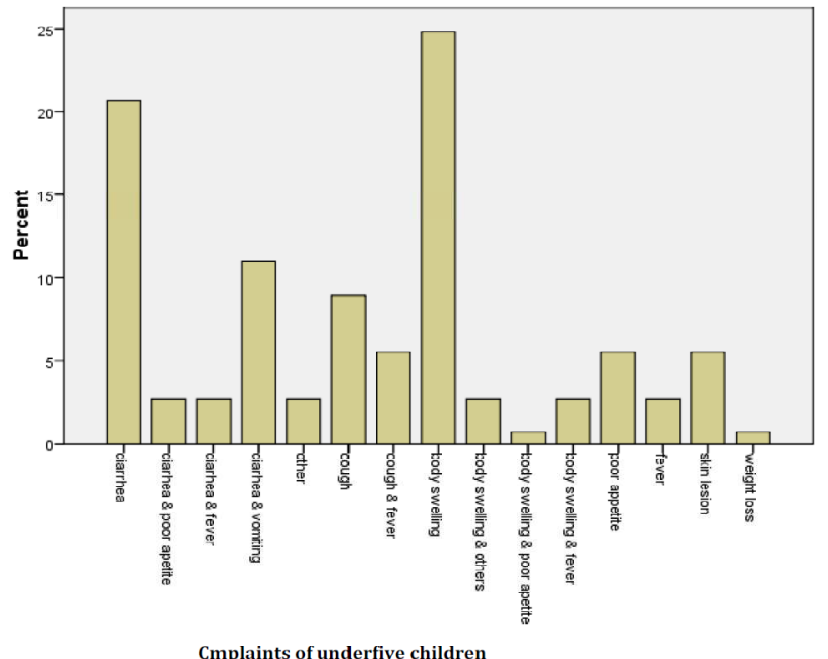

Figure 1. The most common complaints at the time of admission for under five children malnutrition in the study area.

\subsection{Associated Factors with under Five Children Malnutrition}

\subsubsection{Socio-Demographic Characteristics}

Age group was considered for the 145 admitted cases, most of under five children admitted with malnutrition (41.4\%) were 13-24 months old, while children aged 3759 monthsoldwere the least affected (3.4\%). Males were more affected from the admitted under five children (53.1\%) when compared with females (46.9\%) as indicated on Table 1.

Table 1. Socio-demographic characteristics of malnutrition in under five children for one-year retrospective study in FHRH.

\begin{tabular}{lll}
\cline { 1 - 1 } Sociodemographic characteristics & Frequency & $\begin{array}{l}\text { Proportion for } \\
\text { malnutrition (\%) }\end{array}$ \\
\cline { 1 - 2 } Less than 6 & 16 & 11 \\
$6-12$ & 36 & 24.8 \\
$12-24$ & 60 & 41.4 \\
$24-36$ & 28 & 19.3 \\
$36-59$ & 5 & 3.4 \\
Sex & & \\
Males & 77 & 53.1 \\
Females & 68 & 46.9 \\
\hline
\end{tabular}

\subsubsection{Breast Feeding History}

Most of the affected children with malnutrition (61.4\%) were having an average breast feeding duration of about 12-24 months, but significant number of these malnourished children (22\%) were also having breast feeding duration of $<12$ months at all (Table 2).

Table2. Breast feeding duration of the under five children affected with malnutrition in this study.

\begin{tabular}{llll}
\hline $\begin{array}{l}\text { Breast feeding } \\
\text { duration (in } \\
\text { months) }\end{array}$ & Frequency & $\begin{array}{l}\text { \% proportion for } \\
\text { malnutrition }\end{array}$ & Cumulative \% \\
\hline$<12$ & 32 & 22.1 & 22.1 \\
$12-24$ & 89 & 61.4 & 83.4 \\
$>24$ & 12 & 8.3 & 91.7 \\
\hline
\end{tabular}




\subsubsection{Number of Feedings per Day}

The highest proportion $(57.24 \%)$ of under five children affected with malnutrition in this study were being fed for less than 3 times a day and only a smaller group (12.4\%) were being fed for more than 3 times a day (Table 3 ).

Table 3. Number of feedings per day for under five children affected with malnutrition in this study.

\begin{tabular}{llll}
\hline $\begin{array}{l}\text { Number of } \\
\text { feedings per day }\end{array}$ & Frequency & $\begin{array}{l}\text { \% proportion for } \\
\text { malnutrition }\end{array}$ & Cumulative \% \\
\hline Less than 3 times & 83 & 57.24 & 57.24 \\
3 times & 44 & 30.3 & 87.54 \\
$\begin{array}{l}\text { Greater than } 3 \\
\text { times }\end{array}$ & 18 & 12.4 & 100 \\
\hline
\end{tabular}

\subsection{Degree of Malnutrition in Under five Children}

Among the malnourished children detected in this study (145), about 93(64.1\%) were detected for wasting/acute malnutrition and about $36(24.8 \%)$ were affected with severe wasting or severe acute malnutrition (SAM) as shown on Table 4.

Table 4. Degree of wasting/acute malnutrition detected in under five children during the study period.

\begin{tabular}{llll}
\hline $\begin{array}{l}\text { Weight-for-Height } \\
\text { (Percentile) }\end{array}$ & Frequency & $\begin{array}{l}\text { \% } \\
\text { malnourished }\end{array}$ & Cumulative \% \\
\hline$\geq 90$ & 52 & 35.9 & 35.9 \\
$80-90$ & 16 & 11 & 46.9 \\
$70-80$ & 41 & 28.3 & 75.2 \\
$<70$ & 36 & 24.8 & 100 \\
Total & 145 & 100 & 100 \\
\hline
\end{tabular}

Data on the measurement of mid upper arm circumference (MUAC) to determine the level of wasting/acute malnutrition in under five malnutrition, about $30.34 \%$ showed moderate wasting and about $61.38 \%$ of them showed severe wasting/SAM as indicated on Figure 2 below. This degree however is higher than the degree determined by the above weight-for-height procedure.

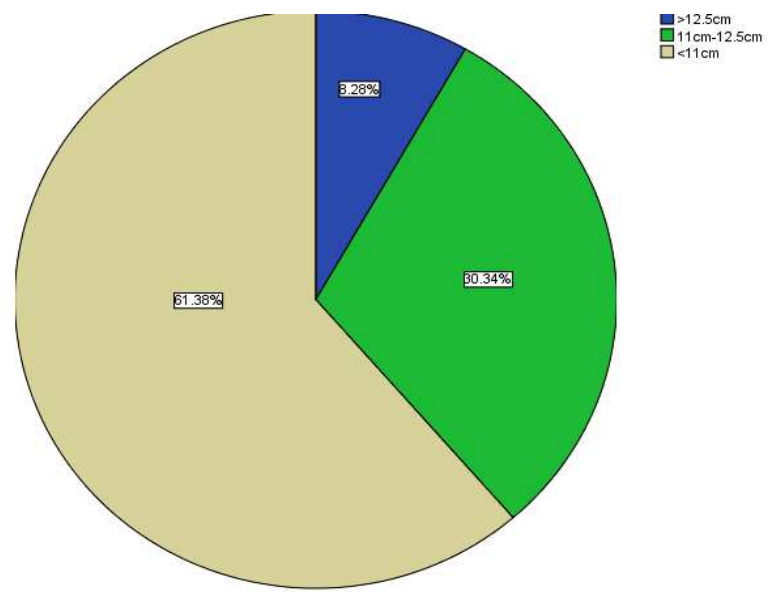

Figure 2. A diagram showing the level of wasting by using MUAC in under five children during the study period (Note: Normal $>$ or equal to $12.5 \mathrm{~cm}$, Moderate wasting for $11-12.5 \mathrm{~cm}$ and Severe wasting for $<11 \mathrm{~cm}$ ).
Among the malnourished children, about 133(91.7\%) were detected for stunting(chronic malnutrition) and about $65(44.8 \%)$ were affected with severe stunting as shown on Table 5.

Table 5. Degree of stunting detected in under five children during the study period.

\begin{tabular}{llll}
\hline $\begin{array}{l}\text { Height-for-Age } \\
\text { (Percentile) }\end{array}$ & Frequency & \% malnourished & Cumulative \% \\
\hline$\geq 95$ & 12 & 8.3 & 8.3 \\
$90-95$ & 24 & 16.6 & 24.8 \\
$85-90$ & 44 & 30.3 & 55.2 \\
$<85$ & 65 & 44.8 & 100 \\
Total & 145 & 100 & 100 \\
\hline
\end{tabular}

Most of these malnourished children were underweight $141(97 \%)$ among which more than half $(53.1 \%)$ were having a Harvard curve of weight-for-age ratio $<60 \%$, which signifies the high burden and severity of the condition at presentation (severe underweight) as shown on Table 6.

Table 6. Harvard curve distribution of weight-for-age for under five children affected with malnutrition in FHRH during the study period.

\begin{tabular}{llll}
\hline $\begin{array}{l}\text { Harvard } \\
\text { Curve/Weight-for- } \\
\text { Age (Percentile) }\end{array}$ & Frequency & $\begin{array}{l}\text { \% } \\
\text { malnourished }\end{array}$ & $\begin{array}{l}\text { Cumulative } \\
\%\end{array}$ \\
\hline$\geq 80$ & 4 & 2.8 & 2.8 \\
$60-80$ & 64 & 44.1 & 46.9 \\
$<60$ & 77 & 53.1 & 100 \\
Total & 145 & 100 & 100 \\
\hline
\end{tabular}

\subsection{Haematological Investigations in Under five Malnourished Children}

Among the malnourished children admitted (145), more than $2 / 3^{\text {rd }}$ of them $(69.6 \%)$ were anemic (hemoglobin level $<$ or equal to $10 \mathrm{~g} / \mathrm{dl}$ )and out of which $8(5.5 \%)$ were severely anemic and were requiring transfusion at the time of admission. The red blood cell size of malnourished under five children was also investigated as shown below on Table 7.

Table 7. Haematological values for under five malnourished children in FHRH during the study period.

\begin{tabular}{llll}
\hline Hematological values & Frequency & \% & Cumulative \% \\
\hline Hemoglobin level $(\mathrm{g} / \mathrm{dl}):$ & & & \\
$\leq 4$ & 8 & 5.5 & 5.5 \\
$5-7$ & 57 & 39.3 & 44.8 \\
$8-10$ & 36 & 24.8 & 69.7 \\
$>10$ & 44 & 30.3 & 100 \\
RBC size(in femtolitres): & & & \\
$\geq 80$ & 49 & 33.8 & 33.8 \\
$<80$ & 80 & 55.2 & 89 \\
Not done & 16 & 11 & 100 \\
\hline
\end{tabular}




\subsection{Therapeutic Management of Malnutrition and Conditions at Discharge for the Under five Malnourished Children}

The under five malnourished children were treated with therapeutic feedings, vitamins including folic acid, Vitamin A, vitamin D, and antibiotics like amoxacillin, ampicillin \&gentamycin and others either in alone or in combination. The therapeutic efficacy of different management options undertaken was not compared as combination therapies were given in most of the cases. However, the overall therapeutic responses and condition at discharge were recorded as improvement (about 67\%), left against medical advice (25\%), dead $(2.76 \%)$ and no change at all (5.52\%) as shown on the Figure 3 below.

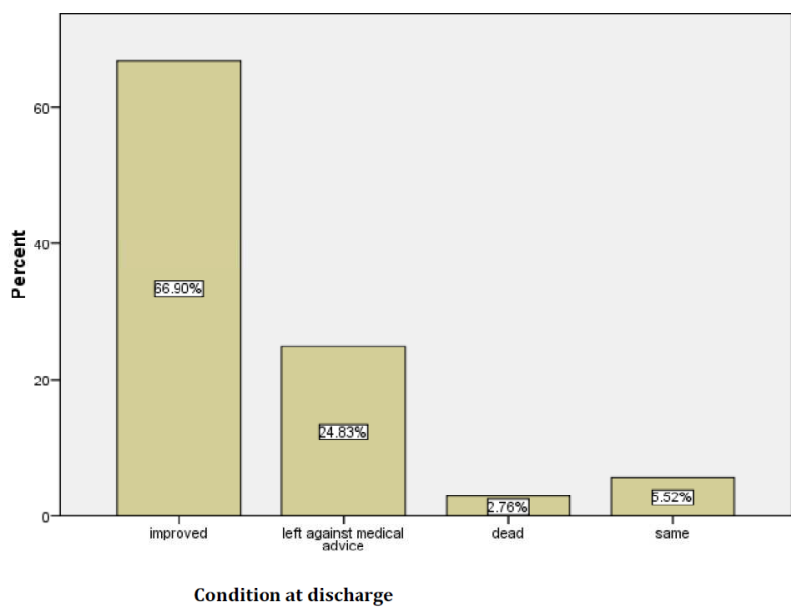

Figure 3. Therapeutic responses and conditions at discharge for the under five malnourished children in FHRH during the study period.

\section{Discussion}

The proportion of malnutrition from the under five admitted children in hospital settings and the major age groups (12-24 months old age) as $41.4 \%$ being affected in this study was high and in concordance with similar studies done in Ethiopia[11]. The higher proportion of malnutrition in children aged 12-24 months could be explained by the existence of next pregnancies and subsequent child neglection for breast feeding by the mothers which will expose the child to be malnourished. Most of the cases of malnutrition in under five children in this study were presenting with generalized body swelling supporting the more prevalence of kwashiorkor (hungry baby syndrome or neglected baby syndrome) in the study area.

Breastfeeding is a norm in Ethiopia and nearly all the children were breastfed although the duration is almost for less than 2 years. As a global public health recommendation, infants should be exclusively breastfeed for the first 6 months of life to achieve optimal growth, development and health. To meet their evolving nutritional requirements, infants should receive nutritionally adequate and safe complementary foods while breastfeeding continues for up to two years of age or beyond [12].
It is possible that bias could occur with the design in this study (especially the data to include the controls was not found) but the associated characteristics of malnutrition and degree of malnutrition in the detected cases were revealed and compared. Despite such shortcomings, this study indicates that many families in the study area are living in poverty, malnutrition and lack of child health-care awareness. We conclude that in order to minimize the prevalence of malnutrition in low socio-economic communities such as in West-Gojjam, it is imperative that parents are encouraged to ensure that their infants are breastfed exclusively for the first six months of life, and breastfed up to the age of two years while including complementary foods which will enable the child to remain healthy perhaps beyond the breast feeding period.

The level/degree of stunting/chronic malnutrition observed in this study is much higher than that of wasting/acute malnutrition( $91.7 \%$ vs $64.1 \%$, respectively). This could be explained by poor socio-economic ad demographic background of families including household resources, number of household children, parental education and maternal nutritional knowledge, as key determinants of chronic child malnutrition $(2,5,6,11)$.

The use of appropriate therapeutic combinations for malnourished children in this study showed improved conditions at discharge following admission. The result of this study also indicates the importance of public health intervention in creating awareness and increasing the knowledge level of the society towards nutritional requirements and the needs of non-delayed therapeutic facilities from the nearby health facilities in children in general and below the age of five years in particular.

\section{Conclusions}

The findings of this study indicated that the proportion of malnutrition in the under five children from a one year retrospective data is high and there appears to be malnutrition proportion differences with factors like sociodemographic characteristics, and inappropriate infant and young child feeding practices.The level/degree of stunting/chronic malnutrition observed in this study was higher than that of wasting/acute malnutrition which probably indicates that the parents of children come to health facilities after at a much delayed time. The use of appropriate therapeutic combinations for malnourished children in this study showed improved conditions at discharge following admission. To reduce under five childhood malnutrition in the area, the following recommendations were forwarded:

- Due emphasis should be given in improving the knowledge and practice of parents on appropriate infant and young child feeding practices and appropriate child health care measures.

- Governmental and non-governmental organizations should be involved to alleviate this alarming situation and possible active interventions should be undertaken 
as soon as possible.

- Future research has to be conducted with more appropriate study designs.

\section{Acknowledgements}

The authors want to gratitude goes to Bahir Dar University/College of Medicine and Health Sciences and Felegehiwot Referal Hospital for facilitating to undertake this study. The authors gratitude also goes to their families.

\section{References}

[1] World Health Organization (2000). Report on malnutrition worldwide, WHO, Geneva.

[2] Stuart, G. and Rafael, F. (2001): Assessing the food and nutrition situation in rural Guatemala.

[3] United Nations Save the Children/UNICEF(1999). Plan of operation in cooperation with Ethiopian government 19941999.

[4] Administrative committee on coordination/sub-committee on nutrition(2000). $4^{\text {th }}$ Report on World nutrition situation, ACC/SCN,UN.
[5] Zewditu, G. (2001). Review of the status of malnutrition and trends in Ethiopia. Ethiop. J Health Dev., 15(2): 55-74.

[6] Gugsa, Y. (2000). Malnutrition among children in southern Ethiopia: levels and risk factors. Ethiop. $J$ Health Dev.,14( 3): 283-292.

[7] Southern Nations Nationalities and Peoples/SNNP(2003). House hold health survey,Awassa, Ethiopia.

[8] Central Statistical Authority (1993). Report on the National Rural Nutrition Survey. Core Module, National Nutritional Surveillance System, Bulletin No 113. Addis Ababa, CSA, Ethiopia. 1993.

[9] Haidar, J., Demissie, T. (1999). Nutrition Situation in Ethiopia.The South African Journal of Clinical Nutrition, 89(2):181-183.

[10] National Center for Health Statistics/World Health Organization(1995). Technical Report,NCHS/WHO, Geneva.

[11] Timotewos, G. (1999). The association of children's nutritional status to maternal education in Zigbaboto, Guragie zone, Ethiopia.Ethiop. J. Health Dev.,13 (1) 1: 5561

[12] Africa Bureau results package (1998): Nutrition, Draft paper. 